\title{
Molecular pathogenesis of virus infections
}

\author{
by M.C. Horzinek \\ Institute of Virology, Veterinary Faculty, de Uithof, Yalelaan 1,3584 CL Utrecht (The Netherlands)
}

Summary. Although a very wide range of viral diseases exists in vertebrates, certain generalizations can be made regarding pathogenetic pathways on the molecular level. The presentation will focus on interactions of virions and their components with target cells. Using coronaviruses as examples the changes in virulence have been traced back to single mutational events; recombination, however, is likely to be an alternative mechanism by which virus-host interactions (e.g. the cell-, organ- or animal species-spectrum) can dramatically change. Receptor molecules are essential for the early interactions during infection and some ot these have been identified. Events in the target cell and the host organism are discussed, and wherever possible, aspects of virus evolution and cooperation between infectious agents are highlighted.

Key words. Virulence; tropism; receptors; cytopathogenicity.

\section{Introduction}

With increasing knowledge about viral genes and their functions, in-depth study of the pathogenesis of virus diseases has become feasible and, in fact, fashionable. However, to write even a superficial review on this subject in general terms is not an easy task. I shall therefore discuss pathogenetic mechanisms using coronaviruses as examples - the family of viruses we have studied in Utrecht since 1978. I will allow for side-steps, however, when illustration is needed and when no pertinent studies have been done with coronaviruses. The interested reader may find more information on the molecular aspects of pathogenesis in the proceedings of a recent symposium devoted to this subject ${ }^{30}$.

I should like to start by defining two terms which, in my opinion, are frequently used in a wrong context: pathogenicity and virulence. I shall designate a virus as pathogenic to describe the detrimental effect it may exert on a host species (e.g. rabies virus is pathogenic for man but apathogenic for frogs). In contrast, virulence refers to the disease-producing potential of a specific strain of a pathogenic virus (e.g. the Flury low egg passage strain of rabies virus is avirulent for dogs but still virulent for kittens).

Epidemiologists have observed that diseases which emerge as new entities are only initially of a highly virulent nature (e.g. canine parvovirus enteritis in 1978); the virulence then gradually lessens until symptomatology becomes slight or absent (e.g. classical swine fever today). In view of this tendency it is obvious that viruses which have reached a stage of commensalism are the 'fittest survivors' since their transmission is guaranteed by an unimpaired behavioral pattern of their hosts. Upon closer examination, however, also viruses which have been labeled as innocuous to their hosts do induce pathology (e.g. lactic dehydrogenase virus of mice may cause an age-dependent polioencephalomyelitis ${ }^{23}$ ). The sudden emergence of new disease agents has lost much of its enigmatic character since some molecular mechanisms have become known. Influenza virus owes its name to the Florence epidemic in 1357 which was believed to have been caused by influentia coeli (celestial influence); it is now one of the bestunderstood pathogens.

Coronaviruses cause respiratory, enteric and generalized infections and disease in animals and man. Also neurologic symptoms have been observed, either under natural conditions e.g. in mouse hepatitis virus (MHV) type 4 infections, in the wake of an infection with feline infectious peritonitis virus (FIPV) or as a laboratory artefact - after intracerebral inoculation of some MHV mutants. The MHV-rodent model has been extensively studied to understand the mechanisms involved in virus induced chronic demyelination.

\section{Target cell specificity: virus tropism}

\section{The virion surface}

Coronaviruses enter the susceptible cell after adsorption to specific receptors by either membrane fusion or endocytosis
- the decisive experiment still needs to be done. Interaction of the viral surface protein - the club-shaped peplomer protruding from the envelope - with a receptor molecule on the cytoplasmic membrane is a prerogative for this process. Evidence has accumulated that already this earliest event during the infection process may determine its outcome. Tropism for glial cells with resulting demyelination has been found in vivo and confirmed in vitro for the JHM strain of MHV type $4^{21}$. Evidence was presented that mutations in the gene coding for the peplomer glycoprotein result in attenuation of neurovirulence ${ }^{4,6}$. It was possible to protect mice by passive immunization using virus-neutralizing monoclonal antibodies. Characterization of neutralization escape mutants obtained with the aid of monoclonal antibodies has shown that resistance at one epitope (E2B) resulted in changes also in another epitope (E2C) and vice versa. This simultaneous acquisition of resistance at multiple epitopes has been interpreted in terms of conformational changes in the polypeptide chain triggered by a single point mutation ${ }^{4}$. In addition, the mutants had lost the important biological property of infect ing neurons. Virus replication was found only in oligodendrocytes, the cell species responsible for providing the myelin sheaths for axons; wild-type MHV-4 infects both cell species. Using the hepatotropic strain A59 of MHV, we have arrived at similar conclusions. Whereas the wild type strain kills mice rapidly, the temperature-sensitive mutant ts 342 is of an attenuated phenotype. It causes chronic demyelinating disease characterized by infection of oligodendrocytes and astrocytes, accompanied by widespread inflammatory reactions in the white matter. We were able to isolate three independent revertants of ts 342 with biological properties indistinguishable from those of the wild type strain. Again it appears that attenuation has resulted from single point mutations ${ }^{17,38}$. A single amino acid substitution in the surface glycoprotein has also been found responsible for the attenuation of neurovirulence in rabies virus 5 .

Single stranded RNA viruses have a predicted mutation rate as high as $10^{-4}-10^{-5}$, which has been experimentally confirmed for coronaviruses ${ }^{4}$. With virus yields of about one hundred progeny infectious units per cell many mutants may be expected to arise during infection of an organism. Apart from resulting in an attenuated phenotype for the individual, these mutants may display novel cell tropisms - also for cells of another animal species. When examining porcine, canine and feline coronaviruses belonging to the same antigenic cluster we found no quantitative nor qualitative differences at the level of the individual polypeptides ${ }^{10}$. However, changes in the host range may also be the consequence of recombinational events. Recombinations have first been shown to occur in the MHV system ${ }^{22}$ but may be more common than anticipated. We have recently compared the nucleotide and derived amino acid sequences of porcine transmissible gastroenteritis virus (TGEV) with those of feline 
infectious peritonitis virus (FIPV) at the peplomer level. Surprisingly, in an amino-terminal stretch of 274 residues, amino acid homology did not exceed $30 \%$ whereupon a sudden transition to a domain of $94 \%$ homology was found $^{11}$. One may speculate that TGEV, which multiplies readily in carnivore cells, has recombined with an autochthonous feline coronavirus, thereby acquiring pathogenicity for the new host.

In some virus-cell systems, cleavage of surface glycoproteins is a prerequisite for infectivity. In paramyxoviruses it is the fusion protein, in orthomyxoviruses the hemagglutinin protein which needs to be proteolytically processed. Cells lacking the relevant enzymes produce noninfectious progeny in a single round of infection ${ }^{16,19}$. Cell culture grown Sendai virus did not cause lung pathology in mice unless it had been treated with trypsin before inoculation. Mutants which possessed a fusion protein cleavable only by chymotrypsin did not produce pneumonia even when activated by this enzyme; only a single round of replication occurred in the organ which lacks the relevant enzyme ${ }^{33}$. These examples show that organ-specific enzymes may permit multiple rounds of infection resulting in organ pathology. However, the enzymes may also be contributed by co-infecting bacteria, as has been shown recently ${ }^{34}$. When mice were exposed to the appropriate combination of strains of influenza virus and Staphylococcus aureus, the animals came down with fatal disease whereas each of the agents inoculated alone caused no significant changes. In view of these findings the concept of 'secondary' bacterial infections may need some adjustment, and antibiotic treatment of influenza pneumonia may not be so bizarre after all.

Also in some coronaviruses proteolytic cleavage of the surface glycoprotein by host cell proteases has been observed. Fusion ensues in infected cells in vivo and in vitro and may be an important virulence determinant. Trypsin treatment was shown to enhance plaque formation and cell fusion of an enteropathogenic bovine coronavirus ${ }^{32}$. Direct evidence for a correlation between cleavage and infectivity, however, has not been presented.

\section{The cell receptor}

The receptor molecules have been identified for several viruses and evidence is accumulating that they serve an essential function in the economy of the cell - otherwise they would probably have been lost from the membrane surface during evolution. Identification of this function has been elusive, however, in most cases. The well-known genetic resistance of some strains of inbred mice to infection with MHV has been found to correlate with the absence of a virus-binding membrane molecule. This structure, probably a protein, was present on the membranes of cells from susceptible and semi-resistant mice and probably represents the MHV receptor ${ }^{2}$.

Mouse-human cellular hybrids have been studied for localizing the receptor of picornaviruses on the human genome; the receptor for poliovirus was found to be coded by a gene on chromosome $19^{25}$. From enzymatic digestion studies it appeared that picornaviruses do not all use the same receptor and that unrelated viruses may share the same receptor.

Binding of vesicular stomatitis virus (VSV) could be entirely inhibited by phosphatidylserine, a common component of cell membranes; this observation may explain the extremely wide host cell range of this rhabdovirus ${ }^{31}$. Rabies virus, another representative of this family, uses acetylcholine receptors for adsorption to neuromuscular junctions, which is compatible with the neurotropic nature of this pathogen ${ }^{20}$. In cell culture, however, rabies virus also infects non-neural cells, which indicates that it may utilize other receptors as well. Monoclonal antibodies are now being widely employed for receptor identification.
Erythrocyte receptors have been studied in hemagglutinating viruses since red blood cells are an abundant source of plasma membrane; the significance of these receptors in relation to the virus-eclipsing structures is not clear, since erythrocytes do not play a role in the pathogenesis of most diseases (e.g. encephalomyocarditis of mice).

Complementarity between the three-dimensional shapes of the viral and the cellular structures is presumably the underlying principle of the interaction. In influenza virus the spikelike hemagglutinin glycoprotein is responsible for initial binding, and a cleft or pocket close to the top of the trimeric molecular aggregate serves as a receptacle for sialoligosaccharides on the cell surface. It is the receptor that generally permits a virus to enter a cell and if replication and amplification occurs, the cell is termed permissive; in an organism, the probability of a virus encountering a permissive cell is small most cell types are non-permissive for a given virus ${ }^{35}$. Also, the receptor density on the surface of a permissive cell may vary with its cycle ${ }^{26}$. For picornaviruses it has been shown that receptors indeed determine the host range ${ }^{24}$; for other viruses, the replication block must occur at a later stage. Parainfluenzaviruses, for example, attach to neuraminic acid-containing receptors; since glycolipids and glycoproteins containing neuraminic acid abound in vertebrate cell membranes the adsorption/penetration process lacks the specificity required to explain the restrictions in host range and tissue tropism of paramyxoviruses ${ }^{29}$.

Cooperation between infectious agents in the pathogenesis of a disease is of special interest in those cases where fulfillment of Koch's postulates has been elusive. In addition to the proteolytic cleavage of the surface glycoprotein discussed above, examples can be given where cell surface receptors for one virus appear after infection with another virus; EpsteinBarr virus was proven to bind to and penetrate into cells lacking the homologous receptor after these cells had been infected with Sendai virus ${ }^{15}$. Baby hamster kidney cells (BHK-21) which are normally refractory to infection with MHV-A59 became susceptible after infection with influenza virus. The (uncleaved) hemagglutinin was shown to provide the receptor on the apical cell surface which recognized the sialic acid residues on the superinfecting coronavirus ${ }^{7}$.

\section{Events in the target cell}

The disease produced by a virus is not necessarily the sum of the cytopathological events. Also viruses non-cytopathogenic in tissue culture do produce disease. In many of these cases immunological mechanisms have been found responsible for the damage caused by the infection; some of these will be discussed later.

The permissiveness, i.e., the degree to which a virus may enter a cell, replicate in it and emerge from it can be restricted at any point in the infectious cycle. Because synthesis of viral components is entirely dependent upon cellular capacities, sharing of molecular functions has evolved. As with many parasite-host relationships, competition often results when shared resources are available in only limited amounts. After adsorption to the cell membrane, enveloped viruses may follow two pathways to have their genome enter the cytoplasmic compartment. In paramyxoviruses, for example, the viral envelope fuses with the cytoplasmic membrane and the contents of the former are released. Alternatively, the virus may utilize the endocytotic pathway and may become internalized into endosomes which then are acidified by proton pumps. At a specific $\mathrm{pH}$, fusion occurs again (between the viral envelope and the endosomal membrane) and the viral genome is released. This pathway is used by e.g. toga-, rhabdo- and orthomyxoviruses. In the latter, the hemagglutinin molecules undergo a conformational change which may bring the adjacent membranes into closer contact. The 
fatty acid chains covalently bound to fusogenic membrane proteins probably destabilize the membrane locally; indeed, deacylated hemagglutinin was found to be devoid of fusogenic activity. The detailed mechanism of fusion, however, is still unknown.

A well-known feature following infection is the ability of many viruses to inhibit the synthesis of cellular macromolecules. The mechanism of this 'shut-off' has been extensively studied in VSV and recently reviewed ${ }^{14}$. VSV is a negative-stranded virus which contains a polymerase; upon infection, transcription starts and a 50-nucleotide leader RNA is synthesized in addition to $5 \mathrm{mRNA}$ species. The leader RNA, especially its central portion (bases 18-30) has been found responsible for the 'shut-off' of cellular RNA synthesis. This sequence shows a high degree of homology with the lupus Sm protein binding regions of small nuclear RNAs. The earliest detectable effect of a VSV infection is the rapid and complete cessation of the prosessing and assembly of the small nuclear RNAs, and it can be inferred that cellular macromolecular synthesis is inhibited and cell death ensues. It is interesting to note that $\beta 1$-interferon production is not blocked by VSV infection. When VSV involved the ability to compromise RNA processing, the host may have adapted to this intervention by evading the requirement for mRNA splicing: the interferon gene lacks introns ${ }^{36}$.

The cytocidal effect of picornavirus infection has been pinpointed as being the inactivation of a cellular cap-binding protein which is essential for proper interaction between ribosomes and host mRNA. Poliovirus RNA translation is independent of this protein ${ }^{37}$. Also in influenza virus infection cap structures are essential: these are cannibalized from host cell nuclear RNA precursor molecules and used as primers for viral RNA replication and synthesis ${ }^{28}$.

The cooperations between infectious agents as they may occur at the level of adsorption and penetration have been discussed above. Also intracellular processes and their pathogenic consequences may be modified by coinfection. The best known example are the defective interfering (DI) particles as they arise during e.g. high multiplicity passages of many viruses. DI particles are mutants of the 'standard' virus which contain (sometimes extreme) deletions and sequence rearrangements. They usually interfere only homotypically (with the replication of the standard virus) at the level of macromolecular synthesis, probably by competition for a replication-relevant polypeptide whose supply in the cell is limited or for virion proteins. Different populations of DI particles can be generated in one system which may differ in biological properties. In the context of this review it is important to mention that restriction of multiplication of standard virus may result in the development of escape mutants resistant to interference. This virus in turn may generate its own DI particles and the cycle repeats itself ${ }^{9}$. It can be speculated that this mechanism grants an antibody-independent evolutionary advantage to the virus and maintains the labile equilibrium established in persistent infections. In cell culture, but also in experimental animal systems, there are cases where DI particles modify an otherwise lethal infection and establish virus persistence (e.g., VSV, reo-, arena- and togaviruses in rodents). For example, adult mice were completely protected from the lethal effect of intranasally administered Semliki Forest virus, a togavirus, by DI particles. The animals showed no clinical signs and cleared the standard virus from their brains in most instances; after several weeks, however, virus could still be isolated with appeared as virulent as the parental standard virus ${ }^{1}$. It remains to be shown whether DI particle-mediated long term virus persistence may give rise to chronic disease in old age.

Another form of cooperation was thought to be a speciality of plant viruses until recently. Parasitic nucleic acids which are capable of very characteristically modulating the expres- sion of disease symptoms of a virus infection are named viral satellites. In spite of the absence of nucleotide sequence relationships between a viral satellite, its helper virus and its host, the trilateral interaction is highly specific ${ }^{13}$. It now appears that the hepatitis delta virus which causes an acute and severe form of hepatitis in humans must be classified as a satellite of hepatitis B virus. Even more exciting is the observation that the circular RNA of the satellite possesses sequence homologies with plant virus genomes which, together with the viroid character of the delta genome, have led to speculations about a possible plant origin of the animal virus ${ }^{18}$.

\section{The role of the host}

Immunity-independent, genetic factors that determine the outcome of an infection have been identified, e.g. in flavivirus infections of mice $^{8}$; selection of genetically defined lines of chickens resistant to Marek's disease, but not to the Herpes virus infection, has been possible.

The molecular interactions between viral gene products, either virion-borne or exhibited at the surface of an infected cell, and complementary structures from the host's immune system, are designed to thwart progression of the infection through the organism. In many cases, however, the immune reaction has been found to be detrimental for the host. Autoimmune phenomena involving both the humoral and cellular limbs of the immune response have been identified in neurological conditions following infections with e.g. canine distemper virus ${ }^{3}$; invasion of brain tissue is supposed to cause changes in the molecular constitution of myelin and membrane components, making them recognizable as "nonself'

Another mechanism involves 'molecular mimicry' - the coincidental similarity between viral and host antigenic determinants, with antibodies recognizing both. Such a crossreactivity was first reported between measles virus and myelin basic protein ${ }^{36}$ and more examples have become known meanwhile. It remains to be shown whether the molecular similarity is indeed responsible for an autoimmune disease condition - the mere comparison of sequences is not sufficient to draw such a conclusion.

The most complex form of autoimmune phenomena involves anti-idiotypic antibodies - those directed against the antigencombining site of an immunoglobulin molecule. These antibodies carry an 'internal image' of the original antigen. In the reovirus/mouse model, anti-idiotypic antibodies have been shown to bind to the viral receptor on the cytoplasmic membrane in a manner similar to virions ${ }^{27}$. Thus impairment of function or even cell injury may occur in tissues distant from those infected as a result of the antiviral immune response and its regulation through the idiotype/anti-idiotype network.

Of all coronavirus infections, feline peritonitis is probably the most enigmatic. In the ascitic fluid and in serum of diseased cats, high concentrations of IgG are found, part of which is antibody formed in reaction to FIPV. Also extremely high-titering neutralizing antibody has no apparent protective value since more seropositive animals with higher titers are detected in catteries where FIP had been a problem; high antibody titers are regarded as diagnostic and prognostic clues. The condition is fatal in the majority of cases. In experimentally infected cats antibody formation started 8-13 days before death and was accompanied by the appearance of circulating immune complexes followed by complement depletion ${ }^{12}$. It is our impression, however, that the immunological phenomena are directed also against host antigens. Attempts to protect cats against this infection by different vaccine preparations have all been unsuccessful. 
Acknowledgment. I should like to thank Willy Spaan for helpful critical comments.

1 Atkinson, P.J., Barrett, A.T.D., and Dimmock, N.J., Defective interfering viruses: modulators of infection. Microbiol. Sci. 2 (1985) $1-7$.

2 Boyle, J.F., Weismiller, D. G., and Holmes, K. V., Genetic resistance to mouse hepatitis virus correlates with absence of virus-binding activity on target tissues. J. Virol. 61 (1987) $185-189$

3 Dal Canto, M.C., and Rabinowitz, S. G., Experimental models of virus-induced demyelination of the central nervous system. Annls Neurol. II (1982) 109-127.

4 Dalziel, R.G., Lampert, P.W., Talbot, P.J., and Buchmeier, M.J., Sitespecific alteration of murine hepatitis virus type 4 peplomer $\mathrm{gly}$ coprotein $\mathrm{E} 2$ results in reduced neurovirulence. J. Virol. 59 (1986) 463-471.

5 Dietzschold, B., Wunner, W. H., Wiktor, T.J., Lopez, A. D., Lafon, M., Smith, C. L., and Koprowski, H., Characterization of an antige nic determinant of the glycoprotein that correlates with patho genicity of rablies virus. Proc. natn. Acad. Sci. USA 80 (1983) $70-74$

6 Fleming, J.O., Trousdale, M.D., El-Zaatari, F.A.K., Stohlman, S.A., and Weiner, L.P., Pathogenicity of antigenic variants of murine coronavirus JHM selected with monoclonal antibodies. J. Virol 58 (1986) 869-875

7 Fuller, S.D., von Bonsdorff, C.-H., and Simmons, K., Cell surface influenza haemagglutinin can mediate infection by other animal viruses. EMBO J. 4 (1985) 2475-2485.

8 Groschel, D., and Koprowski, H., Development of a virus-resistant inbred mouse strain for the study of innate resistance to arbo-B viruses. Arch. ges. Virusforsch. 17 (1965) 379-391.

9 Horodyski, F.M., and Holland, J.J., Reconstruction experiments demonstrating selective effects of defective interfering particles of mixed populations of vesicular stomatitis virus. J. gen. Virol. 65 (1984) 819-823.

10 Horzinek, M.C., Lutz, H., and Pedersen, N. C., Antigenic relationships among homologous structural polypeptides of porcine, feline and canine corona viruses. Infect. Immun. 37 (1982) 1148-1155.

11 Jacobs, L., de Groot, R., van der Zeijst, B. A. M., Horzinek, M. C., and Spaan, W., Comparison of the peplomer protein sequences of porcine transmissible gastroenteritis virus (TGEV) and feline infectious peritonitis virus (FIPV). Virus Res. (1987) in press.

12 Jacobse-Geels, H.E.L., Daha, M. R., and Horzinek, M.C., Antibody, immune complexes, and complement activity fluctuations in kittens with experimentally induced feline infectious peritonitis. Am. J. vet. Res. 43 (1982) 666-670.

13 Kaper, J.M., and Tousignant, M.E., Viral satellites: parasitic nucleic acids capable of modulating disease expression. Endeavour 8 (1984) 194-200

14 Keene, J.D., Interactions between small viral RNAs of vesicular stomatitis virus and components of cellular gene expression. Microbiol. Sci. 2 (1985) 152-156.

15 Khelifa, R., and Menezes, J., Sendai virus envelopes can mediate Epstein-Barr virus binding to and penetration into Epstein-Barr virus receptor-negative cells. J. Virol. 46 (1983) 325-332.

16 Klenk, H.D., Rott, R., Orlich, M., and Blodhorn, J. Activation of influenza virus by trypsin treatment. Virology 68 (1975) 426-439.

17 Koolen, M.I. M., Osterhaus, A. D. M. E., van Steenis, G., Horzinek, M. C., and van der Zeijst, B. A. M., Temperature sensitive mutants of mouse hepatitis virus strain A59: Isolation, characterization and neuropathogenic properties. Virology 125 (1983) 393-402.

18 Kos, A., Dijkema, R., Arnberg, A.C., van der Meide, P.H., and Schellekens, H., The hepatitis delta virus possesses a circular RNA. Nature 323 (1986) 558-560.

19 Lazarowitz, S. G., and Choppin, P. W., Enhancement of the infectivity of influenza $A$ and $B$ viruses by proteolytic cleavage of the hemagglutinin polypeptide. Virology 68 (1975) $440-454$

20 Lentz, T. L., Smith, A. L., Crick, J., and Tignor, G. H., Is the acetylchoine receptor a rabies virus receptor? Science 215 (1981) 182-184.
21 Lucas, A., Flintoff, W., Anderson, R., Percy, D., Coulter, M., and Dales, S., In vivo and in vitro models of demyelinating diseases: Tropism of the JHM strain of murine hepatitis virus for cells of glial origin. Cell 12 (1977) 553-560.

22 Makino, S., Keck, J.G., Stohlman, S.A., and Lai, M.M.C., High frequency RNA recombination of murine coronaviruses. J. Virol. 57 (1986) 729-737.

23 Martinez, D., Brinton, M. A., Tachorsky, T. G., and Phelps, A.H., Identification of lactate dehydrogenase-elevating virus as the etiological agent of genetically restricted, age-dependent polioencephalomyelitis of mice. Infect. Immun. 27 (1980) 979987

24 McClaren, L. C., Holland, J. J., and Syverton, J. T., The mammalian cell-virus relationship. I. Attachment of poliovirus to cultivated cells of primate and non-primate origin. J. exp. Med. 109 (1959) 475-485.

25 Miller, D. A., Miller, O.J., Dev, V.G., Hashmi, S., Tantravahi, R., Medrano, L., and Green, H., Human chromosome 19 carries a poliovirus receptor gene. Cell 1 (1974) 167-173.

26 Morishma, T., McClintock, P. R., Billups, L. C., and Notkins, A. L., Expression and modulation of virus receptors on lymphoid and myeloid cells: Relationship to infectivity. Virology 116 (1982) 605 618.

27 Noseworthy, J.H., Fields, B. N., Dichter, M. A., Sobotka, C., Pizer, E., Perry, L. L., Nepom, T. J., and Greene, M.I., Cell receptors for mammalian reovirus. I. Syngeneic monoclonal anti-idiotypic antibody identifies a cell surface receptor for reovirus. J. Immun. 131 (1983) 2533-2538

28 Plotch, S.W. J., Bouloy, M, and Krug, R. M., Transfer of 5'-terminal cap of globin mRNA to influenza viral complementary RNA during transcription in vitro. Proc, natn. Acad. Sci. USA 76 (1979) 1618 1622

29 Pringle, C. R:, in: Molecular Basis of Virus Disease. Eds W. C. Russell and J.W. Almond. Cambridge University Press (1987).

30 Russell, W.C., and Almond, J.W. (Eds), Molecular Basis of Virus Disease. Cambridge University Press (1987)

31 Schlegel, R., Tralka, T.S., Willingham, M.C., and Pasta, I., Inhibition of VSV binding and infectivity by phosphatidyiserine: Is phosphatidyl-serine a VSV-binding site? Cell 23 (1983) 639-646.

32 Storz, J., Rott, R., and Kaluza, G., Enhancement of plaque formation and cell fusion of an enteropathogenic coronavirus by trypsin treatment. Infect. Immun. 31 (1981) 1214-1222.

33 Tashiro, M., and Homma, M., Pneumotropism of Sendai virus in relation to protease-mediated activation in mouse lungs. Infect. Immun. 39 (1983) 879-888.

34 Tashiro, M., Ciborowski, P., Reinacher, M., Pulverer, G., Klenk, H.-D., and Rott, R., Synergistic role of staphylococcal proteases in the induction of influenza virus pathogenicity. Virology 157 (1987) 421--430.

35 Task Force Report on Persistent Viral Infections-NIAID. US Dept.Hlth Publ. No.79-1833 (1979).

36 Tavernier, J., Derynck, R., and Fiers, W., Evidence for a unique human fibroblast interferon (IFN- $\beta$ l) chromosomal gene, devoid of intervening sequences. Nucl. Acids Res. 9 (1981) 461-471.

37 Trachsel, H., Sonenberg, N., Shatkin, A. J., Rose, J. K., Keong, K. Berman, J.E., Gordon, E., and Baltimore, D. Purification of a factor that restores translation of vesicular stomatitis virus mRNA in extracts form poliovirus infected HeLa cells. Proc. natn. Acad. Sci. USA 77 (1980) 770-774.

38 van Berlo, M.F., Wolswijk, G., Calafat, J., Koolen, M.J. M., Horzinek, M.C., and van der Zeijst, B.A.M., Restricted replication of mouse hepatitis virus A59 in primary mouse brain astrocytes correlates with reduced pathogenicity. J. Virol. 58 (1986) $426-433$

$0014-4754 / 87 / 11 / 121193-04 \$ 150+0.20 / 0$

C) Birkhäuser Verlag Basel, 1987 